\title{
Emerging role of ER quality control in plant cell signal perception
}

\author{
Hong-Ju Li, Wei-Cai Yang ${ }^{\bowtie}$ \\ State Key Laboratory of Molecular Developmental Biology, Institute of Genetics and Developmental Biology, Chinese Academy \\ of Sciences, Beijing 100101, China \\ \ Correspondence: wcyang@genetics.ac.cn \\ Received December 4, 2011 Accepted December 26, 2011
}

\begin{abstract}
The endoplasmic reticulum quality control (ER-QC) is a conserved mechanism in surveillance of secreted signaling factors during cell-to-cell communication in eukaryotes. Recent data show that the ER-QC plays important roles in diverse cell-to-cell signaling processes during immune response, vegetative and reproductive development in plants. Pollen tube guidance is a precisely guided cell-cell communication process between the male and female gametophytes during plant reproduction. Recently, the female signal has been identified as small secreted peptides, but how the pollen tube responds to this signal is still unclear. In this review, we intend to summarize the role of ER-QC in plants and discuss the recent advances regarding our understanding of the mechanism of pollen tube response to the female signals.
\end{abstract}

KEYWORDS cell-to-cell communication, pollen tube guidance, gametophyte, ER quality control

\section{INTRODUCTION}

Cell-cell communication in multicellular and single-celled organisms often requires the recognition between the secreted peptide signals and their receptors that triggers a cascade of signaling pathways to control the cell behavior. With the identification of receptors and ligands, such cell-tocell signal communication pattern has been shown extensively to exist in multiple plant cell signaling processes. It is likely that a pollen tube receptor is also present to recognize the female signals during pollen tube guidance; such receptors and their downstream signaling pathways have not been revealed. Emerging evidence suggests that the endoplasmic reticulum quality control (ER-QC) plays critical roles in receptor maturation during cell signaling in plants. Interestingly, two recent reports suggest that endoplasmic reticulum (ER) most likely plays a central role in pollen tube response to the female cues in male-female gametophytic interactions during pollen tube guidance ( $\mathrm{Li}$ et al, 2011; Lu et al, 2011). This review is therefore focusing on the emerging role of the ER-QC in pollen tube guidance and other processes in plants.

\section{ER QUALITY CONTROL AND CELL SIGNALING IN PLANTS}

In eukaryotic cells, a considerable fraction of proteins are either secreted to the extracellular space or inserted in the plasma membrane to act as ligands and receptors, respectively. In plants, about $30 \%$ of total proteins enter the secretory process via the ER during or after their synthesis, among which $33 \%$ are trans-membrane proteins (Buck et al., 2007). These proteins are synthesized on ER-bound ribosomes and translocated into the ER lumen, which serves as the first organelle of the secretory pathway for the biogenesis of the trans-membrane or secretory protein (Anelli and Sitia, 2008).

ER is responsible for proper assembly/folding of polypeptides before they are targeted to their functional sites and the elimination of misfolded proteins through ER associated degradation (ERAD) (Vembar and Brodsky, 2008). Therefore, ER serves as a site of quality control for proteins, especially those bound for secretion. The ER-QC in plants, monitoring the folding and glycosylation state of the extracellular domain of receptors, is composed of three systems based on studies in animals and yeasts: BiP-mediated protein retention, thiolmediated retention and calnexin/calreticulin (CNX/CRT) cycle on the glycoproteins (Buck et al., 2007; Li et al., 2009). The 
first one is dependent on BiP-ERdj3-GP96/GRP94 complex, which resembles the cytosolic Hsp70-Hsp40-Hsp90 complex in assisting the folding of the non-native polypeptides by recognizing the exposed hydrophobic patches (Jin et al., 2008). The second one is dependent on the disulfide isomerases (PDIs) and other oxidoreductases which recognize free thiol group and transfer disulfide bond to the nascent proteins. These enzymes prevent the secretion of an unassembled cargo protein with unpaired cysteines (Anelli et al., 2003). The third system is specific to glycoproteins (Ellgaard and Helenius, 2001). The client proteins are $\mathrm{N}$ linked with Glc3Man9GIcNAc2 at the Asn residue in the sequence Asn-X-Ser/Thr (X: any amino acid except proline) by STT3A-containing oligosaccharyltransferase (OST) complex. The oligosaccharides are recognized by glucosidases I and II (GI and GII) that subsequently trim off two glucose residues. Then Glc1Man9GIcNAc2 of the glycoprotein is bound by ER membrane-bound CNX or luminal CRT for folding. If the glycoprotein is folded correctly, the terminal Glc is removed by GII and the protein is then released from the CNX or CRT chaperone. If the protein is not folded properly, the terminal Glc is again added by UDP-glucose:glycoprotein glucosyltransferase (UGGT) that distinguishes between folded and unfolded proteins, then the glycosylated protein again gets back to the CNX/CRT cycle until it is properly folded in the ER. The properly folded protein now is ready for secretion, and the misfolded protein will be retro-translocated out of the ER to cytosol where it is degraded by the ERAD (Fig. 1). Although the basic mechanism of the ER-QC is conserved in different cell types and organisms, different subsets of ER-QC components show specificity for different, even closely related, substrates.

The maturation of signaling factors has been extensively studied in animals and yeast, and recently in plants. In human cells, the chaperones in the ER, like CRT, ERP57 and BiP, associate with unassembled subunits or folding intermediates to regulate subunit stability, assembly and expression of receptor complex (Wanamaker and Green, 2007). The ER chaperones are involved in different biological processes with specific substrates to control the response to extracellular stimuli. For example, accumulating evidences suggest that the ER chaperones are involved in cell-cell communication by regulating the receptor maturation. In animals, the folding of Toll receptors and the bradykinin receptor is dependent on ER chaperones during innate immunity (Nakamura et al., 2001; Randow and Seed, 2001). The ER-QC has also been found to function in multiple biological processes in plants. Leucinerich repeat receptor-like kinases (LRR-RLKs) represent the largest group of receptor kinases in plants and act in a wide range of signaling processes in development, stress response and plant-microbe interaction in plants (Diévart and Clark, 2004; Torii, 2004). The LRR-RLKs contain a conserved intracellular kinase domain and a variable extracellular LRR domain (Saijo, 2010). Ligands of the LRR-RLKs such as BRASSINOSTEROID-INSENSITIVE1 (BRI1), FLAGELLIN SENSITIVE2 (FLS2), elongation factor Tu receptor (EFR), XA21, and CLAVATA1 (CLV1), have been identified to be steroid hormones, microbe-derived and plant cell-derived peptides, respectively (Torii, 2004; Boller and Felix, 2009). All these receptors are under the ER-QC surveillance, indicating an indispensible role of the ER-QC in cell signaling in plants.

\section{ER QUALITY CONTROL IN POLLEN TUBE GUIDANCE}

Emerging evidence suggests that the ER-QC also plays a role in male-female gametophytic interaction in Arabidopsis. In higher plants, sperm cells have lost their mobility during evolution, and thus are delivered to the ovule where the egg cell sits by a tip growing tubular structure-the pollen tube formed by the male gametophyte (pollen grain). The pollen tube travels from stigma through the stylar and transmitting tissues via unidirectional tip growth, and turns about $90^{\circ}$ to leave the transmitting tract toward the ovule. This redirection of growth is guided by signals from the ovule. Genetic evidences suggest that the female gametophyte (embryo sac) within the ovule emits attracting signals (Hülskamp et al., 1995). Although the attractants in Arabidopsis remain to be determined, the female attractants in maize (ZmEA1) and Torenia fournieri (TfLUREs) have been identified (Márton et al., 2005; Okuda et al., 2009). These signaling molecules are cysteine-rich polypeptides secreted by the synergid cells of the embryo sac. A concentration gradient of these signaling molecules is likely formed to guide the pollen tube (Dresselhaus and Márton, 2009; Márton and Dresselhaus, 2010). However, very little is known about how pollen tubes perceive and respond to these signals.

In a genetic screen for mutants that disrupt pollen tube guidance, we identified a mutant, pollen defective in guidance 1 (pod1), in which the pollen tubes fail to target the micropyle of the ovule, leading to male sterility (Li, et al., 2011). In pod1 mutant, the growth and morphology of the pollen tube appear to have no difference from that of the wild-type. However, the mutant pollen tubes either are unable to target the micropyle and wandering on the funiculus, or are able to target but unable to enter the micropyle of the ovule, and consequently they grow on the integument surface (Fig. 2). In both cases, the pod 1 tube turns to the ovules and grows on funiculus successfully. This indicates that mutant pollen tube loses its ability to perceive or respond to the female attractants at the micropyle, indicating that the pod 1 tube is defective in micropylar guidance. Often, when the mutant tube fails to enter the ovule, a second wild-type tube is approaching and enters the ovule successfully. This, together with its male transmission defect, indicates that pod1 mutation specifically affects pollen response to the female cue.

$P O D 1$ encodes a novel protein with unknown function. Subcellular localization and functional studies showed that 

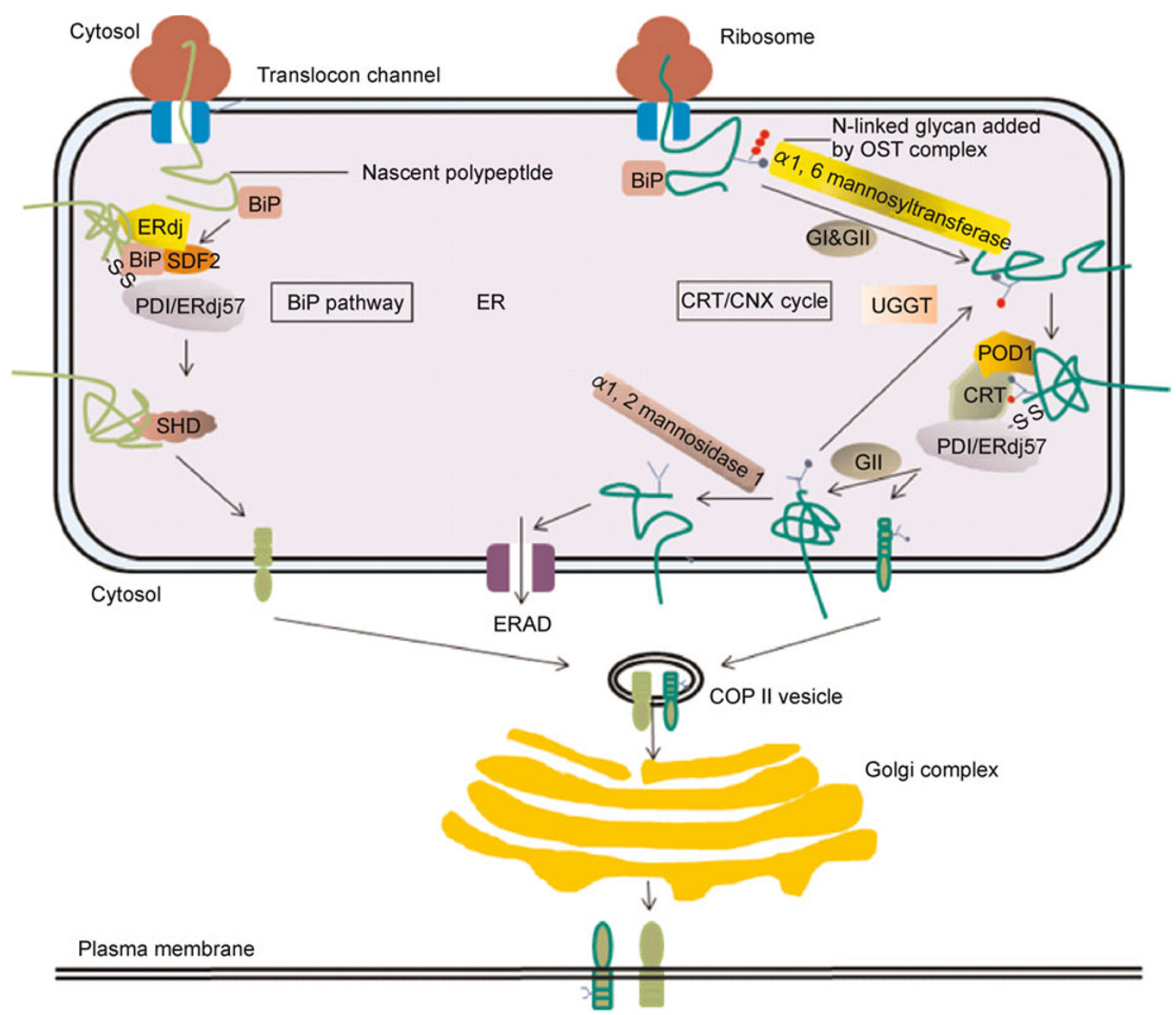

Figure 1. The ER-QC of membrane receptors in plants. The ER is the organelle of synthesis and maturation of nascent polypeptides entering the secretory pathway, including plasma membrane receptors. The newly synthesized polypeptide receptors on the ribosome enter the ER by the translocon complex. The ER contains multiple chaperones, co-chaperones and enzymes that assist the folding/assembly of receptors and retain the misfolded/unassembled proteins in the ER for degradation. This is a quality control (QC) process. Similar to that in animals and yeast, the ER-QC in plants is also composed of three systems: BiP, CNX/CRT and thiol-mediated systems. BiPs recognize the hydrophobic motifs of the nascent polypeptides; CNX/CRT is in charge of the folding of the polypeptides undergoing glycosylation processing; the protein disulfide isomerases (PDI) transfer disulfide bond to the nascent proteins. These three pathways work coordinately to ensure the high folding fidelity of receptors and other signaling factors. The mature receptors are transported into Golgi by COP II complex. The misfolded proteins are finally transported into the cytosol for degradation by the ERAD mechanism. After further glycan modification in Golgi, the functional receptors are transferred to the plasma membrane where they perceive the extracellular signals. ERAD, ER associated degradation.

POD1 is an ER-resident co-chaperone that is not identified previously. Yeast two hybrid screen and bimolecular fluorescence complementation analysis showed that POD1 physically associates with the calcium-binding ER protein calriticulin 3 (CRT3), not with CRT1 or CRT2 (Li et al., 2011). Further analysis also showed that POD1 likely plays a role in ER protein retention. Expression of dominant-negative version of $P O D 1, P O D^{R R R>A A A}$ or $P O D 1^{\triangle N L S}$ causes the mislocalization of the ER marker protein. Consistently, pollen tubes expressing the two mutant versions of $P O D 1$ are not only defective in micropylar pollen tube guidance but also in morphology. This data suggest that POD1 is likely a novel ER chaperone which acts via its interaction with CRT3 in pollen tube guidance. How does an ER protein control pollen tube guidance? Two possible scenarios may exist: (1) POD1 may control the maturation or folding of pollen factors (e.g. receptors) involved in the perception or transduction of female guidance cues in the ER; (2) POD1 might modulate $\mathrm{Ca}^{2+}$ dynamics in the ER to control the cytosolic $\mathrm{Ca}^{2+}$ gradient by interfering with the calcium-binding capacity of CRT3. $\mathrm{Ca}^{2+}$ 


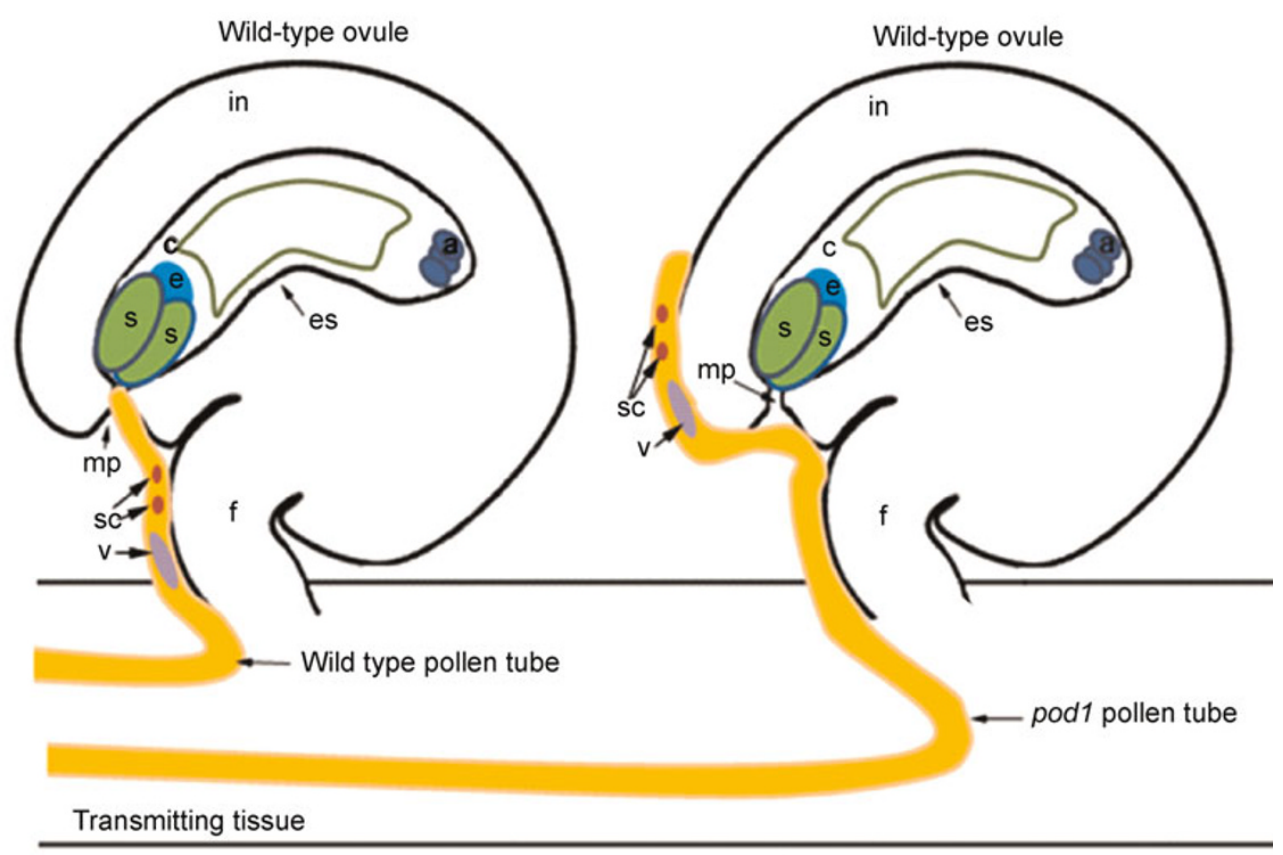

Figure 2. Schematic representation of micropylar response of pod1 pollen tube in Arabidopsis. The embryo sac embedded in the ovule is composed of two synergid cells, an egg cell, a central cell and three antipodal cells. In the wild-type pistil, the wild-type and the pod1 pollen tube containing two sperm cells and one vegetative nucleus grow in the transmitting tissue and further on the integument. The wild-type pollen tube enters the micropyle opening directly and releases the sperm cells into the embryo sac. One sperm fertilizes the egg cell to develop into the embryo; and another sperm fertilizes the central cell to develop into the endosperm. This is called double fertilization process. The pod1 pollen tube travels on the funiculus and further on the integument, but fails to enter the micropyle, leading to male sterility. a, antipodal cell; c, central cell; e, egg cell; es, embryo sac (female gametophyte); $\mathbf{f}$, funiculus; in, integument; $\mathbf{m p}$, micropyle; s, synergid cell; sc, sperm cell; v, vegetative nucleus.

is a well-known signal molecule indispensible for the directional polar growth of the pollen tube (Malhó and Trewavas, 1996). In general, oscillatory $\mathrm{Ca}^{2+}$ gradient is common for growing tips (Cole and Fowler, 2006). This suggests that calcium alone is not sufficient to initiate a change in growth direction, but rather it is required for polar growth.

Indeed, loss-of-function mutations in cyclic nucleotidegated channel CNGC18, whose expression in bacteria results in a time- and concentration-dependent accumulation of cellular $\mathrm{Ca}^{2+}$, impair pollen tube growth (Frietsch et al., 2007). It was also reported that glutamate receptor-like proteins (GLRs) function as $\mathrm{Ca}^{2+}$ channels to regulate the cytosolic $\left[\mathrm{Ca}^{2+}\right]_{\text {cyt }}$ in pollen tubes (Michard et al., 2011). More recently it was shown that the ER-localized $\mathrm{K}^{+}$transporters, cation/ proton exchangers $(\mathrm{CHX})$, are essential for the pollen tube growth out of the transmitting tract. Pollen tubes lacking both $\mathrm{CHX} 21$ and $\mathrm{CHX} 23$ genes grow normally within the transmitting tissues, but fail to turn and leave the transmitting tract ( $\mathrm{Lu}$ et al, 2011). Interestingly, the chx21chx23 double mutant pollen tube also fails to target to the micropyle in the semi-in vitro assay. This indicates that both the funicular and micropylar guidance responses are impaired in chx2chx23 pollen tubes. Together, it suggests that ion homeostasis is important for polen tube growth. It remains to be elucidated how such changes in cellular ion dynamics would lead to changes in growth direction of the pollen tube. It is not known whether POD1 plays a role in $\mathrm{CHX} 21$ and $\mathrm{CHX} 23$ folding in the ER. Nevertheless, it is plausible to speculate that POD1 might also play a role in the ER-QC of ER-localized ion transporter via modulating CRT3 activity during the pollen tube response to the female cue. Currently, such possibility is being investigated.

\section{ER QUALITY CONTROL IN PLANT IMMUNE RESPONSE}

In host-pathogen interaction, formation and maturation of several receptors have been identified to be dependent on the ER chaperones. The innate immune response is dependent on recognition of pathogen-associated molecular pattern (PAMP) by a series of receptors known as pattern recognition receptors (PRRs). EFR, FLS2 and XA21 are well studied PRRs. How PRRs are processed in the ER and how the mature proteins are transported to plasma membrane are under intensive studies. 
In Arabidopsis, FLS2 and EFR share high structural similarity as the LRR XII family members. They work as the receptor of pathogens flagellin and EF-Tu, or their derived peptides flg22 and elf18 in host immune response, respectively (Gómez-Gómez and Boller, 2000; Zipfel et al., 2006; Jeworutzki et al., 2010). FLS2 and EFR activate the downstream defense signaling pathway to enhance plant resistance against pathogens. The maturation of pathogenrecognition receptor EFR, but not FLS2, has been shown to be dependent on the ER quality control components CRT3, UGGT, Glla, GII , STT3A and stromal-derived factor 2 (SDF2). Genetic screens have identified a series of mutants that show impaired EFR accumulation and loss of sensitivity to elf18 peptide. Surprisingly, FLS2 accumulation is not affected, nor its mediated microbe response or affected to a less extent. These mutants are loss-of-function mutants of

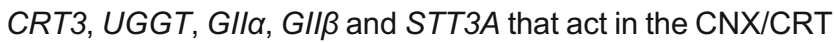
cycle (Li et al., 2009; Lu et al., 2009; Nekrasov et al., 2009; Saijo et al., 2009). The HDEL receptor family member (ERD2b) is also required for EFR accumulation through regulating the ER retention of CRT3 (Li et al., 2009). ERD2b is localized on Golgi and binds the K/HDEL motif of ER-escaped resident proteins and retrieves them back to the ER. Among three CRT members in plant, CRT1 and CRT2 are shown to be involved in general ER chaperone network, but CRT3 is associated with immune response to bacterial PAMP elf18 (Christensen et al., 2010). In tobacco, CRT2 and CRT3 are required for the formation of induced receptor-like kinase (IRK), a plasma membrane localized LRR receptor-like kinase functioning in immune response to tobacco mosaic virus. In the CRT-silenced plants the accumulation of IRK is reduced, but the PM receptor RING4-Citrine is not affected (Caplan et al., 2009). This indicates that CRTs are necessary for the folding or accumulation of IRK by a substrate-specific mechanism. The STT3A and STT3B are subunit isoforms of the Arabidopsis oligosaccharyltransferase involved in protein glycosylation. Loss of both STT3A and STT3A resulted in gametophyte developmental defect (Koiwa et al., 2003), suggesting they are important for gametophyte development. The N-glycosylation of EFR by STT3A in the ER plays a key role for its accumulation and ligand recognition during plantpathogen interaction at the plasma membrane. Proper accumulation of EFR also requires chaperone SDF2ERdj3B-BiP complex in the ER (Nekrasov et al., 2009). Arabidopsis SDF2, an ER-QC component, is required for EFR accumulation as well. In sdf2 mutant, EFR is retained in the ER and finally degraded (Schott et al., 2010). This complex is also required for the formation of FLS2 but to a less extent (Nekrasov et al., 2009). Interestingly, salicylic acid-induced, but EFR-independent immune response requires UGGT and STT3A, but not CRT3, suggesting the existence of another ER-QC component that substitutes the role of CRT3 in this process (Saijo et al., 2009). The stability and processing of the pathogen $X_{0 o}$ recognizing receptor $\mathrm{XA21}$ in rice is dependent on the physical interaction with BiP3 (Park et al., 2010). BiP3 overexpression decreases the XA21 stability and compromises the XA21-mediated immunity but not the general defense response or brassinolide-induced response. These studies indicate that different chaperones of the ERQC system recognize specific substrates, consistent with the specific mutant phenotype in these chaperone genes.

\section{ER QUALITY CONTROL OF DEVELOPMENTAL REGULATORS}

In cell-cell communication during plant meristem development, the maturation of receptors is also ER chaperone dependent. Glucose-regulated protein 94 (GRP94) is an ER resident protein as a counterpart of the cytoplasmic heat shock protein 90 (Hsp90). SHEPHERD (SHD) in Arabidopsis is an ER resident GRP94 chaperone and shd mutant shows pleiotropic phenotypes including meristem differentiation and pollen tube elongation (Ishiguro et al., 2002). CLV3 is a secreted peptide protein in the extracellular space controlling the meristem signaling pathway through the CLV1-CLV2 receptor complex. In the shd mutant, the effect of CLV3 overexpression was abolished. And further phenotypic and genetic analysis also shows that SHD is required for the function of CLV complex during meristem maintenance. In addition, shd pollen tubes fail to elongate in the styles which indicates shd also affects the formation of another protein, receptor or complex required for pollen tube growth in the style. Although the molecular mechanism of STT3A and STT3B is still unclear, it is conceivable that the maturation of a receptor or secretory signal factor essential for the gametophyte development is dependent on these two chaperones (Koiwa et al., 2003). The disulfide isomerase PDIL2-1 has been shown to be involved in embryo sac development (Wang et al., 2008). In addition to the role of pollen tube response, it is also shown that POD1 functions in the early embryo development (Li et al., 2011). These indicate that ERQC also plays key roles in multiple development processes in plant.

\section{ER QUALITY CONTROL OF MUTANT AND UNASSEMBLED RECEPTORS}

The mutant, immature receptors and unassembled receptor complex are retained in the ER by the ER-QC system for further processing or delivered to ERAD for degradation. The Arabidopsis BRI1 is a well-studied brassinosteroid receptor at plasma membrane (Li and Chory, 1997). Two signalcompetent mutant variants bri1-9 and bri1-5 are retained in the ER by CRT3, CNX, UGGT, thiol-mediated system, $\alpha-1,6-$ mannosyltransferase and BiPs (Jin et al., 2007; Hong et al., 2008, 2009; Jin et al., 2009). Mutations in these ER components either restore or enhance the bri1-9 phenotype in Arabidopsis, suggesting that the ER-QC is critical for the 
folding of the BRI1 receptor. This indicates the ER-QC is a very stringent surveillance system and the imperfectly folded, even functional proteins, are retained in the ER. Consistently, glutamate is an excitatory neurotransmitter in the mammalian nervous system. The level of glutamate receptor (GluR) ion channel on the cell surface is dependent on the ER-QC. Only a small fraction of GluR exists on the plasma membrane and most are retained in the ER (Fleck, 2006). This is a mechanism to retain the immature, unassembled or improperly assembled subunits. The nicotinic Ach receptor (AchR) is an ion channel gated by nicotinic acetylcholine (nAch) and mediates neurotransmission. It is composed of $2 \alpha, \beta, \gamma$ and $\delta$ subunits. Evidence has shown that BiP, ERp57 and CNX associate with newly synthesized AchR subunits by stabilizing and sequestering them to regulate the level of assembled AchR in the ER (Wanamaker and Green, 2007). A conserved motif in the transmembrane domain of the subunits functions in the ER retention of the receptor. The motif is hided in the assembled receptor, but exposed in the unassembled subunits. Through this mechanism, the cells transfer the assembled receptor to the cell surface and retain the unassembled subunits in the ER (Wang et al., 2002; Mei and Xiong, 2003).

\section{PERSPECTIVES}

The ER-QC is a conserved mechanism during the maturation of receptors in multiple intercellular communication processes in eukaryotes. As discussed above, the role of the ER-QC system in plants is expanding. A key question is to identify novel proteins that modulate the ER-QC chaperone specificity in a particular process, such as male-female gametophytic cell interaction-a process essential for sexual reproduction. Our understanding on the mechanism of pollen tube guidance has been focused on the female side and the response of the male gametophyte is to be clarified. The emerging role of the ER-QC in pollen tube guidance requires more investigation into the ER-QC components and new players that modulate the ER-QC specificity. Undoubtedly, these will help us to identify the male receptors and proteins involved in pollen tube response and expand our understanding of the ER-QC in general in plant systems.

\section{ACKNOWLEDGEMENTS}

This study was supported by grants from the Ministry of Science and Technology of China (No. 2007CB947600) and National Natural Science Foundation of China (Grant Nos. 30830063 and 30921003) to W.C.Y.

\section{ABBREVIATIONS}

AchR, acetylcholine receptor; BiP, binding immunoglobulin protein; CRT, calreticulin; CNX, calnexin; ERdj3B, ER DnaJ homologue; PAMP, pathogenesis associated molecular pattern; PDI, protein disulfide isomerase; POD1, pollen defective in guidance 1, a CRT3 interacting protein; SDF2, stromal cell-derived factor 2; SHD, SHEPHERD, an ortholog of GRP94; STT3, oligosaccharyltransferase complex subunit; UGGT, UDP-glucose:glycoprotein glucosyltransferase

\section{REFERENCES}

Anelli, T., Alessio, M., Bachi, A., Bergamelli, L., Bertoli, G., Camerini, S., Mezghrani, A., Ruffato, E., Simmen, T., and Sitia, R. (2003). Thiol-mediated protein retention in the endoplasmic reticulum: the role of ERp44. EMBO J 22, 5015-5022.

Anelli, T., and Sitia, R. (2008). Protein quality control in the early secretory pathway. EMBO J 27, 315-327.

Boller, T., and Felix, G. (2009). A renaissance of elicitors: perception of microbe-associated molecular patterns and danger signals by pattern-recognition receptors. Annu Rev Plant Biol 60, 379-406.

Buck, T.M., Wright, C.M., and Brodsky, J.L. (2007). The activities and function of molecular chaperones in the endoplasmic reticulum. Semin Cell Dev Biol 18, 751-761.

Caplan, J.L., Zhu, X., Mamillapalli, P., Marathe, R., Anandalakshmi, R., and Dinesh-Kumar, S.P. (2009). Induced ER chaperones regulate a receptor-like kinase to mediate antiviral innate immune response in plants. Cell Host Microbe 6, 457-469.

Christensen, A., Svensson, K., Thelin, L., Zhang, W., Tintor, N., Prins, D., Funke, N., Michalak, M., Schulze-Lefert, P., Saijo, Y., et al. (2010). Higher plant calreticulins have acquired specialized functions in Arabidopsis. PLoS ONE 5, e11342.

Cole, R.A., and Fowler, J.E. (2006). Polarized growth: maintaining focus on the tip. Curr Opin Plant Biol 9, 579-588.

Diévart, A., and Clark, S.E. (2004). LRR-containing receptors regulating plant development and defense. Development 131, 251-261.

Dresselhaus, T., and Márton, M.L. (2009). Micropylar pollen tube guidance and burst: adapted from defense mechanisms? Curr Opin Plant Biol 12, 773-780.

Ellgaard, L., and Helenius, A. (2001). ER quality control: towards an understanding at the molecular level. Curr Opin Cell Biol 13, 431-437.

Fleck, M.W. (2006). Glutamate receptors and endoplasmic reticulum quality control: looking beneath the surface. Neuroscientist 12 , 232-244.

Frietsch, S., Wang, Y.F., Sladek, C., Poulsen, L.R., Romanowsky, S. M., Schroeder, J.I., and Harper, J.F. (2007). A cyclic nucleotidegated channel is essential for polarized tip growth of pollen. Proc Natl Acad Sci U S A 104, 14531-14536.

Gómez-Gómez, L., and Boller, T. (2000). FLS2: an LRR receptor-like kinase involved in the perception of the bacterial elicitor flagellin in Arabidopsis. Mol Cell 5, 1003-1011.

Hong, Z., Jin, H., Fitchette, A.C., Xia, Y., Monk, A.M., Faye, L., and Li, J. (2009). Mutations of an $\alpha 1,6$ mannosyltransferase inhibit endoplasmic reticulum-associated degradation of defective brassinosteroid receptors in Arabidopsis. Plant Cell 21, 3792-3802.

Hong, Z., Jin, H., Tzfira, T., and Li, J. (2008). Multiple mechanismmediated retention of a defective brassinosteroid receptor in the endoplasmic reticulum of Arabidopsis. Plant Cell 20, 3418-3429.

Hülskamp, M., Schneitz, K., and Pruitt, R.E. (1995). Genetic evidence for a long-range activity that directs pollen tube guidance in Arabidopsis. Plant Cell 7, 57-64. 
Ishiguro, S., Watanabe, Y., Ito, N., Nonaka, H., Takeda, N., Sakai, T., Kanaya, H., and Okada, K. (2002). SHEPHERD is the Arabidopsis GRP94 responsible for the formation of functional CLAVATA proteins. EMBO J 21, 898-908.

Jeworutzki, E., Roelfsema, M.R., Anschütz, U., Krol, E., Elzenga, J.T., Felix, G., Boller, T., Hedrich, R., and Becker, D. (2010). Early signaling through the Arabidopsis pattern recognition receptors FLS2 and EFR involves Ca-associated opening of plasma membrane anion channels. Plant J 62, 367-378.

Jin, H., Hong, Z., Su, W., and Li, J. (2009). A plant-specific calreticulin is a key retention factor for a defective brassinosteroid receptor in the endoplasmic reticulum. Proc Natl Acad Sci USA 106, 15973-15978.

Jin, H., Yan, Z., Nam, K.H., and Li, J. (2007). Allele-specific suppression of a defective brassinosteroid receptor reveals a physiological role of UGGT in ER quality control. Mol Cell 26, 821-830.

Jin, Y., Awad, W., Petrova, K., and Hendershot, L.M. (2008). Regulated release of ERdj3 from unfolded proteins by BiP. EMBO J 27, 2873-2882.

Koiwa, H., Li, F., McCully, M.G., Mendoza, I., Koizumi, N., Manabe, Y., Nakagawa, Y., Zhu, J., Rus, A., Pardo, J.M., et al. (2003). The STT3a subunit isoform of the Arabidopsis oligosaccharyltransferase controls adaptive responses to salt/osmotic stress. Plant Cell 15, 2273-2284.

Li, H.J., Xue, Y., Jia, D.J., Wang, T., Hi, D.Q., Liu, J., Cui, F., Xie, Q., Ye, D., and Yang, W.C. (2011). POD1 regulates pollen tube guidance in response to micropylar female signaling and acts in early embryo patterning in Arabidopsis. Plant Cell 23, 3288-3302.

Li, J., and Chory, J. (1997). A putative leucine-rich repeat receptor kinase involved in brassinosteroid signal transduction. Cell 90 , 929-938.

Li, J., Zhao-Hui, C., Batoux, M., Nekrasov, V., Roux, M., Chinchilla, D., Zipfel, C., and Jones, J.D. (2009). Specific ER quality control components required for biogenesis of the plant innate immune receptor EFR. Proc Natl Acad Sci U S A 106, 15973-15978.

Lu, X., Tintor, N., Mentzel, T., Kombrink, E., Boller, T., Robatzek, S., Schulze-Lefert, P., and Saijo, Y. (2009). Uncoupling of sustained MAMP receptor signaling from early outputs in an Arabidopsis endoplasmic reticulum glucosidase II allele. Proc Natl Acad Sci U S A 106, 22522-22527.

Lu, Y., Chanroj, S., Zulkifli, L., Johnson, M.A., Uozumi, N., Cheung, A., and Sze, H. (2011). Pollen tubes lacking a pair of $K^{+}$transporters fail to target ovules in Arabidopsis. Plant Cell 23, 81-93.

Malhó, R., and Trewavas, A.J. (1996). Localized apical increases of cytosolic free calcium control pollen tube orientation. Plant Cell 8 , 1935-1949.

Márton, M.L., Cordts, S., Broadhvest, J., and Dresselhaus, T. (2005). Micropylar pollen tube guidance by egg apparatus 1 of maize. Science 307, 573-576.

Márton, M.L., and Dresselhaus, T. (2010). Female gametophytecontrolled pollen tube guidance. Biochem Soc Trans 38, 627-630.

Mei, L., and Xiong, W.C. (2003). Two birds with one stone: a novel motif for ACh receptor assembly quality control. Trends Neurosci 26, 178-181.
Michard, E., Lima, P.T., Borges, F., Silva, A.C., Portes, M.T., Carvalho, J.E., Gilliham, M., Liu, L.H., Obermeyer, G., and Feijó, J.A. (2011). Glutamate receptor-like genes form $\mathrm{Ca}^{2+}$ channels in pollen tubes and are regulated by pistil D-serine. Science 332, 434-437.

Nakamura, K., Zuppini, A., Arnaudeau, S., Lynch, J., Ahsan, I., Krause, R., Papp, S., De Smedt, H., Parys, J.B., Muller-Esterl, W., et al. (2001). Functional specialization of calreticulin domains. J Cell Biol 154, 961-972.

Nekrasov, V., Li, J., Batoux, M., Roux, M., Chu, Z.H., Lacombe, S., Rougon, A., Bittel, P., Kiss-Papp, M., Chinchilla, D., et al. (2009). Control of the pattern-recognition receptor EFR by an ER protein complex in plant immunity. EMBO J 28, 3428-3438.

Okuda, S., Tsutsui, H., Shiina, K., Sprunck, S., Takeuchi, H., Yui, R., Kasahara, R.D., Hamamura, Y., Mizukami, A., Susaki, D., et al. (2009). Defensin-like polypeptide LUREs are pollen tube attractants secreted from synergid cells. Nature 458, 357-361.

Park, C.J., Bart, R., Chern, M., Canlas, P.E., Bai, W., and Ronald, P.C. (2010). Overexpression of the endoplasmic reticulum chaperone $\mathrm{BiP} 3$ regulates XA21-mediated innate immunity in rice. PLoS One 5, e9262.

Randow, F., and Seed, B. (2001). Endoplasmic reticulum chaperone gp96 is required for innate immunity but not cell viability. Nat Cell Biol 3, 891-896.

Saijo, Y. (2010). ER quality control of immune receptors and regulators in plants. Cell Microbiol 12, 716-724.

Saijo, Y., Tintor, N., Lu, X., Rauf, P., Pajerowska-Mukhtar, K., Häweker, H., Dong, X., Robatzek, S., and Schulze-Lefert, P. (2009). Receptor quality control in the endoplasmic reticulum for plant innate immunity. EMBO J 28, 3439-3449.

Schott, A., Ravaud, S., Keller, S., Radzimanowski, J., Viotti, C., Hillmer, S., Sinning, I., and Strahl, S. (2010). Arabidopsis stromalderived Factor2 (SDF2) is a crucial target of the unfolded protein response in the endoplasmic reticulum. J Biol Chem 285, 18113-18121.

Torii, K.U. (2004). Leucine-rich repeat receptor kinases in plants: structure, function, and signal transduction pathways. Int Rev Cytol 234, 1-46.

Vembar, S.S., and Brodsky, J.L. (2008). One step at a time: endoplasmic reticulum-associated degradation. Nat Rev Mol Cell Biol 9, 944-957.

Wanamaker, C.P., and Green, W.N. (2007). Endoplasmic reticulum chaperones stabilize nicotinic receptor subunits and regulate receptor assembly. J Biol Chem 282, 31113-31123.

Wang, H., Boavida, L.C., Ron, M., and McCormick, S. (2008). Truncation of a protein disulfide isomerase, PDIL2-1, delays embryo sac maturation and disrupts pollen tube guidance in Arabidopsis thaliana. Plant Cell 20, 3300-3311.

Wang, J.M., Zhang, L., Yao, Y., Viroonchatapan, N., Rothe, E., and Wang, Z.Z. (2002). A transmembrane motif governs the surface trafficking of nicotinic acetylcholine receptors. Nat Neurosci 5, 963-970.

Zipfel, C., Kunze, G., Chinchilla, D., Caniard, A., Jones, J.D., Boller, T., and Felix, G. (2006). Perception of the bacterial PAMP EF-Tu by the receptor EFR restricts Agrobacterium-mediated transformation. Cell 125, 749-760. 